\title{
New two-step iteration process for solving the semiconductor plasma generation problem with arbitrary $\mathrm{BC}$ in $2 \mathrm{D}$ case
}

\author{
V. A. Trofimov, M. M. Loginova \& V. A. Egorenkov \\ Lomonosov Moscow State University, Russia
}

\begin{abstract}
Using the original two-step iteration process, we develop a conservative finitedifference scheme for the problem of femtosecond pulse propagation in semiconductor under the action of an external electric field. Its advantages consist in its applicability for the problem with non-uniform boundary conditions and it possesses also a property of asymptotic stability. Therefore, it is possible to provide a computation for long interval time without losing the conservatism property. We pay special attention to the calculation of initial function distribution, which are a solution of the set of 2D stationary partial differential equations. We solve this set of equations by using an additional iteration process that is similar to the iteration process applied for the solution of the main problem. Using computer simulation, it is shown that the proposed conservative finite-difference scheme is an effective tool for calculation of complicated regimes of semiconductor characteristic changing. Semiconductor plasma generation under the action of external electric field is investigated.

Keywords: conservative finite-difference scheme, iteration process convergence, femtosecond pulse, semiconductor.
\end{abstract}

\section{Introduction}

Laser radiation interaction with semiconductor is very actual problem. This process accompanies by many nonlinear effects such as optical bistability (OB) [1], for example, and it is governed by both nonlinear equations, which describe an evolution of free-electron concentration and concentration of ionized donors, and Poisson equation for electric field strength, and equation with respect to laser pulse intensity. For computer simulation of these complicated non-stationary 
processes it is necessary to use a finite-difference scheme possessing such characteristics as conservatism, high precision of calculations and asymptotic stability to initial data changing. These requirements are caused by developing of periodic processes for laser pulse interaction with a semiconductor if the OB takes place. As a rule, a period of semiconductor characteristic oscillation is greater than 100 dimensionless units. Moreover, OB occurring leads to appearance of domains with high concentrations of charged particles (electrons and ionized donors) and these domains have sharp boundaries. Another characteristic feature of $\mathrm{OB}$ realization is an explosive absorption of laser energy. Therefore, in certain time moments the free-charged particle concentrations change very fast. It means that the concentrations have big derivative in time.

One of the well-known methods for solving of multi-dimensional equations is the split-step method [2]. However, in [3] we have shown that this method has some disadvantages if we apply it for the solution of the problem under consideration. Therefore, in [4] we proposed and developed a new finitedifference scheme for numerical solution of such kind of the problems. Its main feature consists in using two-step iteration process for solving the set of the corresponding nonlinear equations. This allows us to realize both a conservatism property for finite-difference scheme and its asymptotic stability as well. In the present paper we continue our research for the problem with arbitrary boundary conditions and proposed the additional iteration process for calculation of initial function distributions.

\section{Statement of $2 \mathrm{D}$ problem}

The process of semiconductor plasma generation is described by the following set of 2D dimensionless differential equations [5]:

$$
\begin{gathered}
\frac{\partial n}{\partial t}=D_{x} \frac{\partial}{\partial x}\left(\frac{\partial n}{\partial x}-\mu_{x} n \frac{\partial \phi}{\partial x}\right)+D_{y} \frac{\partial}{\partial y}\left(\frac{\partial n}{\partial y}-\mu_{y} n \frac{\partial \phi}{\partial y}\right)+G(N, n)-R(N, n), \\
\frac{\partial N}{\partial t}=G(N, n)-R(N, n), \frac{\partial^{2} \phi}{\partial x^{2}}+\frac{\partial^{2} \phi}{\partial y^{2}}=\gamma(n-N), \frac{\partial I}{\partial y}+\delta_{0} \delta(N, n) I=0, \\
0<x<L_{x}, 0<y<L_{y}, t>0 .
\end{gathered}
$$

Above the following notations are introduced. Function $n$ denotes a free electron concentration in the conductivity zone of a semiconductor; $N$ is a concentration of ionized donors. Function $\phi$ describes a dimensionless electric field potential. $I$ is the intensity of laser radiation propagating along the $y$ axis. The coordinate $x$ is a coordinate that is transverse to the direction of laser pulse propagation. Variables $x, y$ are dimensionless spatial coordinates, $L_{x}, L_{y}$ denote their maximal values, correspondingly. Variable $t$ denotes a dimensionless time. Coefficients of electron diffusion $D_{x}, D_{y}$ and coefficients of electron mobility $\mu_{x}, \mu_{y}$ are non-negative constants. Parameter $\gamma$ depends, in particular, on the maximal concentration of free charged particles, $\delta_{0}$ denotes maximal value of 
absorption coefficient for laser energy. Dependence of the absorption coefficient $\delta(N, n)$ from charged particle concentrations can be approximated by different ways in dependence on physical experiment conditions. Below we consider the following dependence:

$$
\delta(N, n)=(1-N) e^{-\alpha(1-\beta n)}, \alpha, \beta>0,
$$

which is close to one of the experimental dependencies [1]. The functions $G$ and $R$, describing generation and recombination of free charged particles in the semiconductor, are given by the formulas:

$$
G(N, n)=q_{0} I \delta(N, n), R(N, n)=\frac{N n-n_{0}^{2}}{\tau_{p}},
$$

$n_{0}$ is an equilibrium value of the free electron concentration and ionized donor concentration, corresponding to absence of both the light pulse action on a semiconductor and external electric field. Parameter $\tau_{p}$ characterizes a recombination time of free electrons, $q_{0}$ is a maximal intensity of the incident laser pulse. Its profile is Gaussian one along the x-coordinate:

$$
\left.I\right|_{y=0}=\exp \left(-\left(\frac{x-0.5 L_{x}}{0.1 L_{x}}\right)^{2}\right)(1-\exp (-10 t)) .
$$

Boundary conditions for the set of the equations (1) are written below. They correspond to absence of electric current through semiconductor faces and semiconductor is placed in the external electric field as well:

$$
\begin{gathered}
\left.\left(\frac{\partial n}{\partial x}-\mu_{x} n \frac{\partial \phi}{\partial x}\right)\right|_{x=0, L_{x}}=\left.\left(\frac{\partial n}{\partial y}-\mu_{y} n \frac{\partial \phi}{\partial y}\right)\right|_{y=0, L_{y}}=0, \\
\left.\frac{\partial \phi}{\partial x}\right|_{x=0, L_{x}}=-E_{x},\left.\frac{\partial \phi}{\partial y}\right|_{y=0, L_{y}}=-E_{y} .
\end{gathered}
$$

Initial conditions for the free charged particle concentrations under the external electric field action are written in the following manner:

$$
\begin{gathered}
n(x, y, t=0)=n_{0}(x, y), N(x, y, t=0)=N_{0}(x, y), \\
\phi(x, y, t=0)=\phi_{0}(x, y),\left.I\right|_{t=0}=0,
\end{gathered}
$$

where $n_{0}(x, y), N_{0}(x, y), \phi_{0}(x, y)$ are functions, which are found from the solution of the set of equations (1) in steady-state case and without laser pulse action.

For the problem (1)-(6) the law of charged particles preservation takes place:

$$
Q(t)=\int_{0}^{L_{y}} \int_{0}^{L_{x}}(n(x, y, t)-N(x, y, t)) d x d y=0 .
$$

A difference analogue of the invariant (7) should preserve and this property shouldn't be loosen due to the accumulation of a computing error even for 
calculation on long time interval. So, our aim is to construct the conservative finite-difference scheme with the property of asymptotic stability.

\section{Finite-difference scheme for the problem}

To solve the problem (1)-(6) numerically let us introduce in the domain

$$
\bar{G}=\left\{0 \leq x \leq L_{x}\right\} \times\left\{0 \leq y \leq L_{y}\right\} \times\left\{0 \leq t \leq L_{t}\right\}
$$

the uniform grids in time and space

$$
\begin{gathered}
\Omega=\omega_{x} \times \omega_{y} \times \omega_{t}, \Omega^{\prime \prime}=\omega_{x} \times \omega^{\prime}{ }_{y} \times \omega^{\prime}{ }_{t}, \\
\omega_{x}=\left\{x_{i}=i h_{x}, i=\overline{0, N_{x}}, h_{x}=L_{x} / N_{x}\right\}, \omega_{y}=\left\{y_{j}=j h_{y}, j=\overline{0, N_{y}}, h_{y}=L_{y} / N_{y}\right\}, \\
\omega_{t}=\left\{t_{k}=k \tau, k=\overline{0, N_{t}}, \tau=L_{t} / N_{t}\right\}, \\
\omega_{y}^{\prime}=\left\{y_{j}^{\prime}=(j-0.5) h_{y}, j=\overline{0, N_{y}+1}, h_{y}=L_{y} / N_{y}\right\}, \\
\omega_{t}^{\prime}=\left\{t_{k}^{\prime}=(k+0.5) \tau, k=\overline{0, N_{t}-1}, \tau=L_{t} / N_{t}\right\} .
\end{gathered}
$$

Let's define the mesh functions $n_{h}, N_{h}, \phi_{h}$ on the grid $\Omega$ in the following way: $n_{i j k}=n\left(x_{i}, y_{j}, t_{k}\right), N_{i j k}=N\left(x_{i}, y_{j}, t_{k}\right), \phi_{i j k}=\phi\left(x_{i}, y_{j}, t_{k}\right)$.

Function $I_{h}$ we define on the mesh $\Omega^{\prime \prime}$ shifted additionally on both spatial coordinate $y$ and time coordinate: $I_{i j k}=I\left(x_{i}, y_{j}^{\prime}, t_{k}^{\prime}\right)$.

For brevity, we use the following index-free notations:

$$
\begin{gathered}
f=f_{i j k}, \hat{f}=f_{i j k+1}, \stackrel{0.5}{f}=0.5(f+\hat{f}), \\
f_{i \pm 1}=f_{i \pm 1 j k}, f_{j \pm 1}=f_{i j \pm 1 k}, f_{i \pm 0.5}=0.5\left(f+f_{i \pm 1}\right), f_{j \pm 0.5}=0.5\left(f+f_{j \pm 1}\right), \\
I=I_{i j k}, I_{i \pm 1}=I_{i \pm 1 j k}, \hat{I}=I_{i j+1 k}, \stackrel{0.5}{I}=0.5(I+\hat{I}) .
\end{gathered}
$$

where $f$ is one of the mesh functions: $n_{h}, N_{h}, \phi_{h}$. For the finite-difference scheme construction we use also the following notations:

$$
\begin{gathered}
R=\left(n N-\left(n_{0}\right)^{2}\right) / \tau_{p}, \quad \hat{R}=\left(\hat{n} \hat{N}-\left(n_{0}\right)^{2}\right) / \tau_{p}, \quad \stackrel{0.5}{R}=0.5(R+\hat{R}), \\
G=q_{0} \stackrel{0.5}{I} \delta, \hat{G}=q_{0} \stackrel{0.5}{I} \hat{\delta}, \stackrel{0.5}{G}=0.5(G+\hat{G}), \\
\delta=(1-N) \exp (-\alpha(1-\beta n)), \hat{\delta}=(1-\hat{N}) \exp (-\alpha(1-\beta \hat{n})), \quad \stackrel{0.5}{\delta}=0.5(\delta+\hat{\delta}) .
\end{gathered}
$$

The first and the second differential derivatives are defined in standard way and notated as follows: $f_{x}, f_{\bar{x}}, f_{\bar{x} x}, f_{y}, f_{\bar{y}}, f_{\bar{y} y}, f_{t}$.

In the case under consideration, the property of conservatism of the finitedifference scheme consists in performance of a difference analogue of the conservation law (7) for charged particle concentrations: 


$$
Q\left(t_{k}\right)=\sum_{i=1}^{N_{x}-1} \sum_{j=1}^{N_{y}-1} h_{x} h_{y}\left(n_{i j k}-N_{i j k}\right)=0 .
$$

For numerical solution of the problem (1)-(6) we approximate the initialboundary problem by the set of finite-difference equations. For their resolvability we use two-step iteration process. Below the first step of the iteration process with the boundary conditions is written:

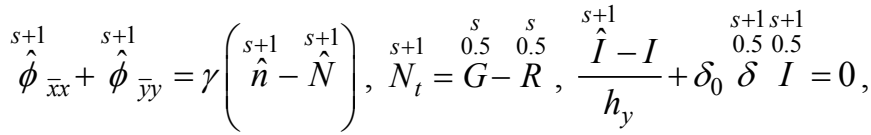

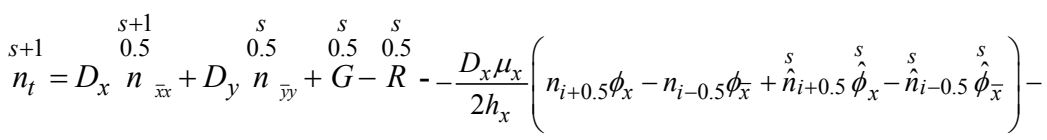

$$
\begin{aligned}
& -\frac{D_{y} \mu_{y}}{2 h_{y}}\left(n_{j+0.5} \phi_{y}-n_{j-0.5} \phi_{\bar{y}}+\stackrel{s}{\hat{n}_{j+0.5}} \hat{\phi}_{y}-s_{j-0.5} \hat{\phi}_{\bar{y}}\right) \text {, } \\
& \left.\hat{\phi}_{y}^{s+1}\right|_{j=0}=\left.\hat{\phi} \hat{\phi}_{\bar{y}}^{s+1}\right|_{j=N_{y}}=-E_{y}, i=0, \ldots, N_{x}, \\
& \left.\stackrel{s+1}{\hat{\phi}_{x}}\right|_{i=0}=\left.\hat{\phi}_{\bar{x}}^{s+1}\right|_{i=N_{x}}=-E_{x}, j=0, \ldots, N_{y}, \\
& \left.\stackrel{s+1}{\hat{n}}\right|_{x=0}=-\left.\mu_{x} \hat{n}_{i+0.5} E_{x}\right|_{i=0},\left.\stackrel{s+1}{\hat{n}} \bar{x}\right|_{i=N_{x}}=-\left.\mu_{x} \hat{n}_{i-0.5} E_{x}\right|_{i=N_{x}}, j=0, \ldots, N_{y} .
\end{aligned}
$$

The second step of the iteration process, written also with the boundary conditions, is:

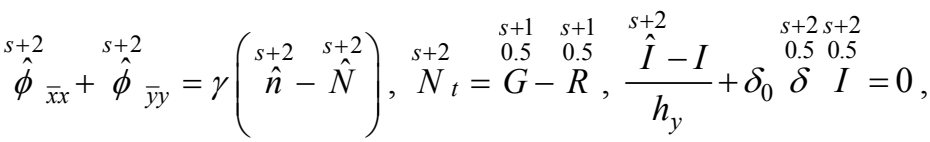

$$
\begin{aligned}
& \begin{array}{lllll} 
& s+1 & s+2 & s+1 & s+1 \\
s+2 & 0.5 & 0.5 & 0.5 & 0.5
\end{array} \\
& n_{t}=D_{x} n \bar{x} x+D_{y} \quad n \bar{y} y+G-R- \\
& -\frac{D_{x} \mu_{x}}{2 h_{x}}\left(n_{i+0.5} \phi_{x}-n_{i-0.5} \phi_{\bar{x}}+\stackrel{n}{n}_{i+0.5} \begin{array}{ccc}
s+1 & s+1 & s+1 \\
\hat{\phi}_{x}-\hat{n}_{i-0.5} & \hat{\phi}_{\bar{x}}
\end{array}\right)- \\
& -\frac{D_{y} \mu_{y}}{2 h_{y}}\left(n_{j+0.5} \phi_{y}-n_{j-0.5} \phi_{\bar{y}}+\hat{n}_{j+0.5}^{s+1} \hat{\phi}_{y}-\hat{n}_{j-0.5}^{+1} \hat{\hat{\phi}}_{\bar{y}}^{s+1}\right), \\
& \left.\stackrel{s+2}{\hat{\phi}}\right|_{y=0}=\left.\hat{\phi}_{\bar{y}}^{s+2}\right|_{j=N_{y}}=-E_{y}, i=0, \ldots, N_{x},
\end{aligned}
$$




$$
\begin{gathered}
\left.\hat{\phi}_{x}^{s+2}\right|_{i=0}=\left.\hat{\phi}_{\bar{x}}^{s+2}\right|_{i=N_{x}}=-E_{x}, j=0, \ldots, N_{y}, \\
\hat{n}_{y}+\left.2\right|_{j=0}=-\left.\mu_{y} \hat{n}_{j+0.5}^{s+1} E_{y}\right|_{j=0},\left.\stackrel{s+2}{\hat{n}} \bar{y}\right|_{j=N_{y}}=-\left.\mu_{y} \hat{n}_{j-0.5} E_{y}\right|_{j=N_{y}}, i=0, \ldots, N_{x}, \\
I_{i 0 k}=\exp \left(-\left(\frac{x_{i}-x_{0.5 N_{x}}}{0.1 L_{x}}\right)^{2}\right)\left(1-e^{-10 t_{k}}\right), k=0, \ldots, N_{t}, i=0, \ldots, N_{x} .
\end{gathered}
$$

As an initial approach for the iteration process, the values of the functions, obtained on the previous time layer, are undertaken:

$$
\stackrel{s=0}{\hat{n}}=n, \quad \stackrel{s=0}{\hat{N}}=N, \quad \hat{\phi}=0 \quad \hat{\phi}, \quad \hat{I}=0 .
$$

The iteration process is stopped if the following inequalities are valid:

$$
\left|\begin{array}{cc}
s+2 & s \\
\hat{n}-\hat{n}
\end{array}\right| \leq \varepsilon_{1}\left|\begin{array}{l}
s \\
\hat{n}
\end{array}\right|+\varepsilon_{2},\left|\begin{array}{cc}
s+2 & s \\
\hat{N}-\hat{N}
\end{array}\right| \leq \varepsilon_{1}\left|\begin{array}{c}
s \\
\hat{N}
\end{array}\right|+\varepsilon_{2},\left|\begin{array}{cc}
s+2 & s \\
\hat{I} & -\hat{I}
\end{array}\right| \leq \varepsilon_{1}|\hat{I}|+\varepsilon_{2}, \quad \varepsilon_{1}, \varepsilon_{2}>0 .
$$

We don't check up an accuracy of the electric field potential in (12) because we use additional iteration process for solving of the Poisson equation.

The finite-difference scheme possesses the second order of approximation on spatial coordinates and on time in the inner grid nodes. The boundary conditions are approximated with the first order. This is caused by the conservatism property validity. Necessity of such boundary conditions approximation was shown in [3] for $1 \mathrm{D}$ case problem.

\subsection{Remark to the Poisson equation solution}

At solving of the Poisson equation concerning the electric field potential (1) we have to construct an additional iteration process in such a way, that we have to solve only the $1 \mathrm{D}$ problem on each of iterations. Taking into account a linearity of this equation we use the split-step method. For this purpose we introduce additional function $F$ on the grid $\bar{\Omega}=\omega_{x} \times \omega_{y}$, which is governed by the problem:

$$
\begin{gathered}
F^{0}=\hat{\phi}, \frac{F^{p+1}-F^{p}}{\bar{\tau}}=F_{\bar{x} x}^{p+1}+F_{\bar{y} y}^{p}-\gamma\left(\begin{array}{c}
s+1 \\
\hat{n}-\hat{N}
\end{array}\right), \\
\frac{F_{\bar{\tau}}^{p+1}-F^{p+1}}{\bar{\tau}}=F_{\bar{x} x}^{p+1}+F_{\bar{y} y}^{p+2}-\gamma\left(\begin{array}{c}
s+1 \\
\hat{n}-\hat{N}
\end{array}\right), i=1, \ldots, N_{x}-1, j=1, \ldots, N_{y}-1,
\end{gathered}
$$

where $\bar{\tau}$ is an iteration parameter.

For an assessment of the iteration process convergence we use the criterion based on the discrepancy assessment:

$$
\Psi=\left|F_{\bar{x} x}^{p+2}+F_{\bar{y} y}^{p+2}-\gamma\left(\begin{array}{c}
s+1 \\
\hat{n}-\hat{N}
\end{array}\right)\right| \leq \varepsilon_{3,} \varepsilon_{3}>0 .
$$


If the solution, obtained on the $p+2$ iteration, satisfies to the criterion (14), then we choose $\hat{\phi} \hat{\phi}=F^{p+2}$.

\subsection{Calculation of the initial function distributions}

Now let's discuss the problem of initial function distribution for the problem (1)(6). If the external electric field is absent then we have the simple initial conditions for the problem: $n_{0}(x, y)=N_{0}(x, y)=n_{0}, \phi_{0}(x, y)=0$. If the external electric field acts only on one of the spatial coordinates $\left(E_{x}=0\right.$ or $E_{y}=0$ ) or if the electron mobility is the same $\left(\mu_{x}=\mu_{y}=\mu\right)$, we can define initial distribution of the functions as [6]: $n_{0}(x, y)=n_{0} e^{\mu \phi}, N_{0}(x, y)=n_{0} e^{-\mu \phi}$ and find the electric field potential $\phi_{0}(x, y)$ as a solution of equation:

$$
\frac{\partial^{2} \phi}{\partial x^{2}}+\frac{\partial^{2} \phi}{\partial y^{2}}=\gamma\left(n_{0} e^{\mu \phi}-n_{0} e^{-\mu \phi}\right) .
$$

However, if the arbitrary boundary conditions or difference between electron mobility coefficients take place, then it is necessary to solve additional problem. Because the laser radiation is absent at the initial time moment, the generation of free charged particles does not occur. Therefore, the following equality $R=0$ is valid. Thus, we obtain the following set of $2 \mathrm{D}$ stationary differential equations:

$$
\begin{gathered}
\frac{\partial^{2} \phi}{\partial x^{2}}+\frac{\partial^{2} \phi}{\partial y^{2}}=\gamma(n-N), N n-n_{0}^{2}=0, \\
D_{x} \frac{\partial}{\partial x}\left(\frac{\partial n}{\partial x}-\mu_{x} n \frac{\partial \phi}{\partial x}\right)+D_{y} \frac{\partial}{\partial y}\left(\frac{\partial n}{\partial y}-\mu_{y} n \frac{\partial \phi}{\partial y}\right)=0,0<x<L_{x}, 0<y<L_{y},
\end{gathered}
$$

with the boundary conditions (5).

For solution of the problem (15) we also construct two-step iteration process, as we done above, using the meshes defined in (8). For example, the finitedifference scheme for the case $D_{x} \geq D_{y}$ is written below. First step of iteration process with boundary conditions is:

$$
\begin{gathered}
\frac{N^{p+1}-N^{p}}{\tilde{\tau}}=-\frac{N^{p} n^{p}-n_{0}^{2}}{\tau_{r}}, i=0, \ldots, N_{x}, j=0, \ldots, N_{y}, \\
\frac{n^{p+1}-n^{p}}{\tilde{\tau}}=\frac{D_{x}}{D_{y}} n_{\bar{x} x}^{p+1}-\frac{D_{x}}{D_{y}} \mu_{x}\left(n_{i+0.5}^{p} \phi_{x}^{p}-n_{i-0.5}^{p} \phi_{\bar{x}}^{p}\right)+ \\
+\boldsymbol{n}_{\bar{y} y}^{p}-\mu_{y}\left(\boldsymbol{n}_{j+0.5}^{p} \boldsymbol{\phi}_{y}^{p}-\boldsymbol{n}_{j-0.5}^{p} \boldsymbol{\phi}_{\bar{y}}^{p}\right)-\frac{N^{p} n^{p}-n_{0}^{2}}{\tau_{r}}, \\
\frac{\phi^{p+1}-\phi^{p}}{\tilde{\tau}}=\boldsymbol{\phi}_{\bar{x} x}^{p+1}+\boldsymbol{\phi}_{\bar{y} y}^{p}-\gamma\left(\boldsymbol{n}^{p+1}-\boldsymbol{N}^{p+1}\right), i=1, \ldots, N_{x}-1, j=1, \ldots, N_{y}-1, \\
\left.\boldsymbol{\phi}_{x}^{p+1}\right|_{i=0} ^{p}=\left.\boldsymbol{\phi}_{\bar{x}}^{p+1}\right|_{i=N_{x}}=-E_{x}, j=0, \ldots, N_{y},
\end{gathered}
$$




$$
\left.n_{x}^{p+1}\right|_{i=0}+\left.\mu_{x} n_{i+0.5}^{p} E_{x}\right|_{i=0}=0,\left.n_{\bar{x}}^{p+1}\right|_{i=N_{x}}+\left.\mu_{x} n_{i-0.5}^{p} E_{x}\right|_{i=N_{x}}=0, j=0, \ldots, N_{y} .
$$

Second step of iteration process with the boundary conditions is the following:

$$
\begin{gathered}
\frac{N^{p+2}-N^{p+1}}{\tilde{\tau}}=-\frac{N^{p+1} n^{p+1}-n_{0}^{2}}{\tau_{r}}, i=0, \ldots, N_{x}, j=0, \ldots, N_{y}, \\
\frac{n^{p+2}-n^{p+1}}{\tau}=\frac{D_{x}}{D_{y}} n_{\bar{x} x}^{p+1}-\frac{D_{x}}{D_{y}} \mu_{x}\left(n_{i+0.5}^{p+1} \phi_{x}^{p+1}-n_{i-0.5}^{p+1} \phi_{\bar{x}}^{p+1}\right)+ \\
+n \frac{p}{\bar{y} y}-\mu_{y}\left(n_{j+0.5}^{p+1} \phi_{y}^{p+1}-n_{j-0.5}^{p+1} \phi_{\bar{y}}^{p+1}\right)-\frac{N^{p+1} n^{p+1}-n_{0}^{2}}{\tau_{r}}, \\
\frac{\phi^{p+2}-\phi^{p+1}}{\tilde{\tau}}=\phi_{\bar{x} x}^{p+1}+\phi_{\bar{y} y}^{p+2}-\gamma\left(n^{p+2}-N^{p+2}\right), i=1, \ldots, N_{x}-1, \\
\left.\phi_{y}^{p+2}\right|_{j=0}=\left.\phi_{\bar{y}}^{p+2}\right|_{j=N_{y}}=-E_{y}, i=0, \ldots, N_{x}, \\
\left.n_{y}^{p+2}\right|_{j=0}+\left.\mu_{y} n_{j+0.5}^{p+1} E_{y}\right|_{j=0}=0,\left.n_{\bar{y}}^{p+2}\right|_{j=N_{y}}+\left.\mu_{y} n_{j-0.5}^{p+1} E_{y}\right|_{j=N_{y}}=0, i=0, \ldots, N_{x},
\end{gathered}
$$

where $\tilde{\tau}$ is an iteration parameter, $\tau_{r}$ is a regularization parameter, which is added for the computation performance enhancing. We use the following initial approach for our calculation:

$$
n^{p=0}=N^{p=0}=n_{0}, \phi^{p=0}=0, i=0, \ldots, N_{x}, j=0, \ldots, N_{y} .
$$

Criteria of the iteration process convergence are chosen in following way:

$$
\begin{aligned}
& \phi_{\bar{x} x}^{p+2}+\phi_{\bar{y} y}^{p+2}-\gamma\left(n^{p+2}-N^{p+2}\right) \mid<\bar{\varepsilon}_{1}, \\
& \frac{D_{x}}{D_{y}} n_{\bar{x} x}^{p+2}-\frac{D_{x}}{D_{y}} \mu_{x}\left(n_{i+0.5}^{p+2} \phi_{x}^{p+2}-n_{i-0.5}^{p+2} \phi_{\bar{x}}^{p+2}\right)+ \\
& +n \bar{y}_{y y}^{p+2}-\mu_{y}\left(n_{j+0.5}^{p+2} \phi_{y}^{p+2}-n_{j-0.5}^{p+2} \phi_{\bar{y}}^{p+2}\right) \mid<\bar{\varepsilon}_{2}, \bar{\varepsilon}_{1}, \bar{\varepsilon}_{2}>0 .
\end{aligned}
$$

For the solution of the Poisson equation we use the Thomas algorithm. It should be also stressed, that the proposed finite-difference scheme (16)-(17) is a conservative one on each of iterations. It could be easily shown that the invariant (9) is valid for each of iterations.

\section{Computer simulation results}

Accuracy of initial condition calculation plays a basic role and has essential influence on the accuracy of the problem (1)-(6) solution. The distributions of free electron concentration, corresponding to calculations of the initial function distributions with various accuracies, are depicted in Fig. 1. As it is follows from 
the Fig. 1, the solution symmetry could be significantly distorted because of calculation error accumulation if the initial function distributions are calculated with low accuracy $\bar{\varepsilon}_{1}=\bar{\varepsilon}_{2}=10^{-2}$.



(a)

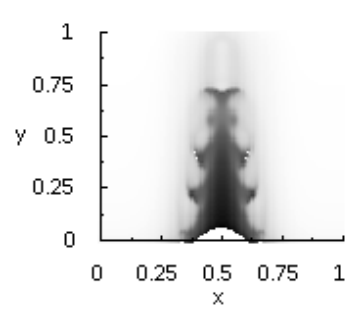

(d)

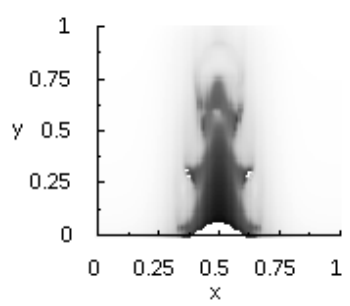

(b)

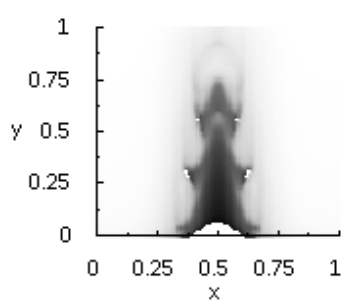

(e)

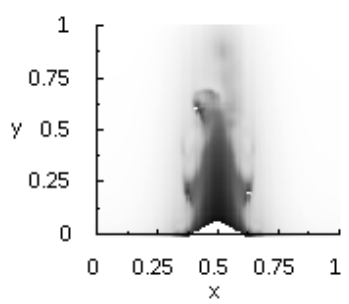

(c)

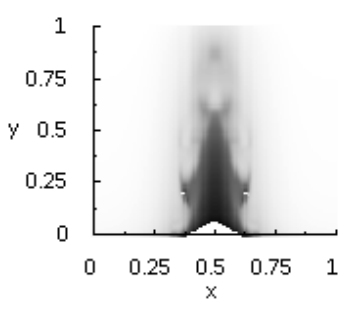

(f)

Figure 1: Free electron concentration distribution obtained for parameters

$$
\begin{aligned}
& \delta_{0}=2, q_{0}=1.5, D_{x}=D_{y}=10^{-5}, \gamma=10^{3}, n_{0}=0.01, \quad \mu_{x}=\mu_{y}=1, \\
& \tau_{p}=1, \quad \xi=3, \psi=2.553, E_{x}=0, E_{y}=8, \quad L_{x}=L_{y}=1 \quad \text { and } \\
& \text { computation parameters } h_{x}=h_{y}=10^{-2}, \tau=5 \cdot 10^{-4}, \quad \tau=10^{-4}, \\
& \bar{\tau}=\tilde{\tau}=10^{-4}, \quad \tau_{r}=10^{-4}, \quad \varepsilon_{1}=10^{-5}, \quad \varepsilon_{2}=10^{-7}, \quad \varepsilon_{3}=10^{-3}, \\
& \overline{\varepsilon_{1}}=\bar{\varepsilon}_{2}=10^{-2} \text { (a), (b), (c) and } \bar{\varepsilon}_{1}=\bar{\varepsilon}_{2}=10^{-4} \quad \text { (d), (e), (f) at time } \\
& \text { moments } t=250 \text { (a), (b), } t=320 \text { (c), (d), } t=500 \text { (e), (f). }
\end{aligned}
$$

However, for $E_{x}=0$ the concentration distribution should possess symmetry along the $x$-coordinate. Moreover, in this case it is impossible to reach a high accuracy of the electric field potential calculation for the problem (1)-(6): value of the discrepancy (16) increases up to 0.7 and couldn't be decreased by means of iteration number increasing. However, if initial function distribution calculates with enough high accuracy $\bar{\varepsilon}_{1}=\bar{\varepsilon}_{2}=10^{-4}$, the discrepancy (16) has the order of magnitude that is less than $10^{-3}$. In this case, the symmetry of the concentration distribution takes place. Therefore, the calculation accuracy of initial electric field potential distribution plays a key role under solving the problem (1)-(6). 
As we stressed above our aim was to develop the finite-difference scheme for solution of the problem under consideration with arbitrary boundary conditions. To illustrate opportunities of developed finite-difference scheme, below computer simulation results made for non-uniform boundary conditions on both spatial coordinates are shown. Moreover, we carry out computing for different electron mobility coefficients for a long time interval without losing the conservatism property. As one can see from Fig. 2, very complicated periodic regimes of semiconductor characteristics changing take place under the action of high intensive laser pulse [7].

We see a strong dependence of the free-electron concentration evolution on the electron mobility relation. If the electron mobility along the x-coordinate is less than its value along the y-coordinate then the "bird-like" structure appears over big time interval (Fig. 2 (j)). If the electron mobility components are the same for both coordinates then a spatial structure, which is similar to helical waves, appears (Fig. $2(\mathrm{k})$ ). Let us notes, that usually such kind of structures takes place if electrons move under the action of magnetic field. However, if the OB takes place and the external electric field acts on the semiconductor then the helical waves are formed.

The last considered case corresponds to a case of domination of the electron mobility along the $\mathrm{x}$-coordinate over the $\mathrm{y}$-component of the electron mobility (Fig. 2 (1)). We see strong asymmetric electron concentration distribution in the first half domain with respect to incident laser pulse. Moreover, under certain relation between the diffusion coefficients one can expect closing the domains with high electron concentration on the right side of central part of the domain.

We have to emphasize one more feature of considered laser pulse interaction with a semiconductor under the OB existence. We see in Fig. 2 that for majority of time moments, the domain with low electron concentration takes place near the semiconductor face, which corresponds to falling of the laser pulse. This domain cannot exist if the external electric field does not act along the y-coordinate in chosen direction.

We see also the high free-electron concentration domain appearance near the ending face of the semiconductor with respect to the laser pulse propagation direction. This is caused exactly by the electric field action directed along the yaxis. Another condition for the low free-electron concentration occurring is certain value of the electric field strength: its magnitude must be enough for the high freeelectron concentration domain appearance near the ending face of the semiconductor.

In the end of this paragraph we stress one more that the computer simulation results were obtained for mesh steps being equal to 0.01 along both spatial coordinates. For spatial structures under consideration this value of mesh steps is sufficient big. Nevertheless, we see stable spatial structures which appearance and evolution is explained physically in the frame-work of both OB existence and the external electric field action as well. So, the developed finite-difference scheme is effective one for computation of such kind of laser pulse interaction with a semiconductor. 


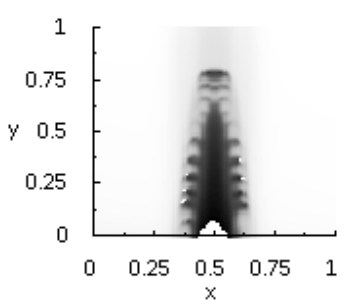

(a)

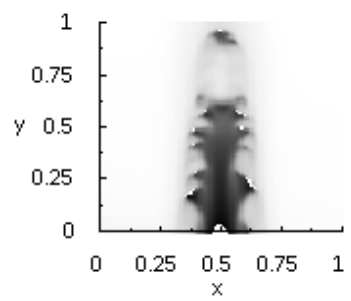

(d)

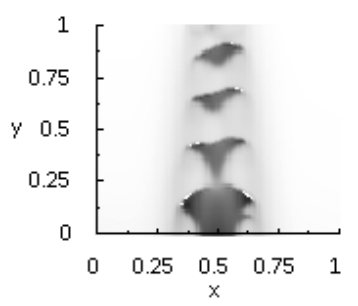

(g)

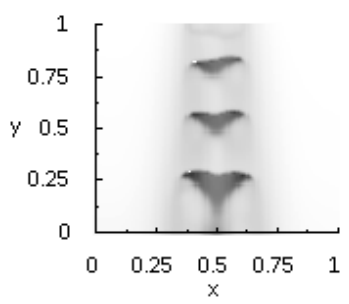

(j)

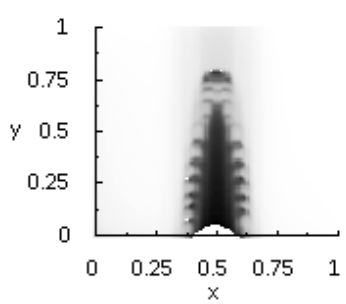

(b)

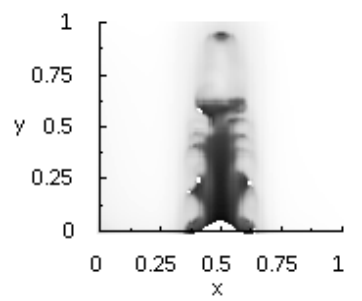

(e)

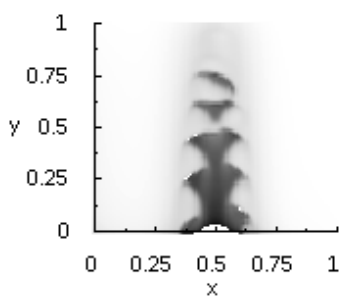

(h)

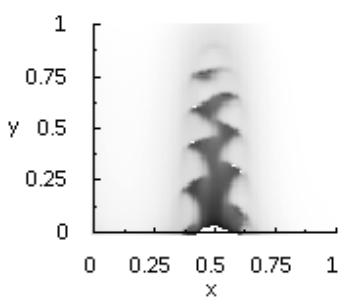

(k)

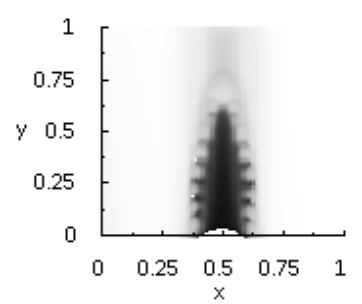

(c)

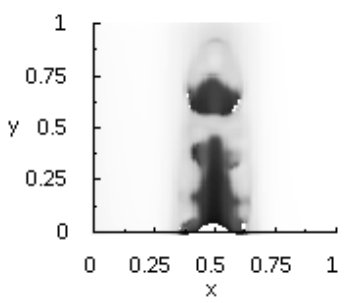

(f)

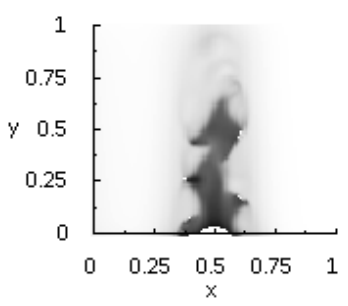

(i)

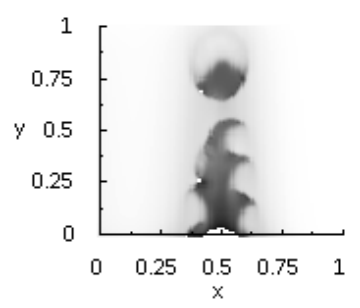

(1)

Figure 2: Free electron concentration distribution calculated for parameters $\delta_{0}=0.5, q_{0}=0.5, D_{x}=D_{y}=10^{-5}, \gamma=10^{3}, n_{0}=0.01, \tau_{p}=1, \quad \xi=3$, $\psi=2.553, E_{x}=8, E_{y}=8, \quad L_{x}=L_{y}=1, \mu_{x}=0.1, \mu_{y}=1$ (a), (d), (g), (j), $\mu_{x}=\mu_{y}=1$ (b), (e), (h), (k), $\mu_{x}=1, \mu_{y}=0.1$ (c), (f), (i), (l) at time moments $t=50$ (a), (b), (c), $t=100$ (d), (e), (f), $t=320$ (g), (h), (i), $t=500$ (j), (k), (l). 


\section{Conclusions}

In the present paper the finite-difference scheme for the problem of plasma generation with arbitrary boundary conditions is proposed on the basis of the twostep iteration process. One of its main advantages consists in property of the asymptotic stability. Computer simulation results show the essential influence of accuracy of initial function distribution calculation on evolution of the free charged particle concentrations. We also demonstrated that the finite-difference scheme developed in the present paper is applicable for calculating of nonlinear complicated processes, which occur in a semiconductor under the action of high intensive laser pulse.

\section{Acknowledgement}

The investigation was made using support of the Russian Science Foundation (Grant № 14-21-00081).

\section{References}

[1] Gibbs H.M. Optical Bistability: Controlling Light with Light, Academic Press, NY, 1985.

[2] Strang G. "On the construction and comparison of differential schemes", SIAM Journal of Numerical Analysis, V.5, N3, pp. 506-517 (1968).

[3] Trofimov V.A., Loginova M.M. "Difference scheme for the problem of femtosecond pulse interaction with semiconductor in the case of nonlinear electron mobility", Journal of Computational Mathematics and Mathematical Physics, V.45, N12, pp. 2185-2196 (2005).

[4] Trofimov, V.A., Loginova, M.M., Egorenkov, V.A., "Influence of external electric field on laser- induced wave process occurring in semiconductor under the femtosecond pulse acting", Proceedings of SPIE , V. 9127, 912709 (2014).

[5] Smith R., Semiconductors, Cambridge University Press, 1978.

[6] Il'in V.P. Difference methods for electrophysics problems, Novosibirsk: Nauka, 1985 (in Russian).

[7] Trofimov V.A., Egorenkov V.A., Loginova M.M. "Helical auto-waves electron-hole plasma in semiconductor induced by femtosecond pulse at presence of external electric field", Proceedings of SPIE, V. 9200, 920004 (2014). 